\title{
Fecal microbiota transplantation derived from Alzheimer's disease mice worsens brain trauma outcomes in young C57BL/6 mice
}

\author{
Sirena Soriano ${ }^{1}$, Kristen Curry ${ }^{2}$, Qi Wang ${ }^{2}$, Elsbeth Chow $^{1}$, Todd Treangen $^{2}$, Sonia Villapol ${ }^{\star 1,3}$ \\ ${ }^{1}$ Department of Neurosurgery and Center for Neuroregeneration, Houston Methodist Research \\ Institute, Houston, TX, ${ }^{2}$ Department of Computer Science, Rice University, Houston, TX, \\ ${ }^{3}$ Department of Neuroscience in Neurological Surgery, Weill Cornell Medical College, NY.
}

*Corresponding author: Department of Neurosurgery and Center for Neuroregeneration, Houston Methodist Research Institute, 6670 Bertner Avenue, Houston, 77030, TX. Phone: (713) 363-9208; email: svillapol@houstonmethodist.org. ORCID ID: orcid.org/0000-0002-6174-4113 


\section{Abstract}

Traumatic brain injury (TBI) cause neuroinflammation, exaggerated immune response, and, consequently, neurodegeneration. The gut microbiome is an essential modulator of the immune system, impacting in the brain. There are not effective treatments for TBI, therefore, modulating the gut microbiome may shed novel therapeutics for the damaged brain. Also, in patients with Alzheimer's disease (AD), the microbiota has been associated with a lack of diversity, which negatively modulates the immune system. This study aimed to determine whether the gut microbiota from $A D$ mice exacerbates neurological deficits after TBI in young mice. For this purpose, we performed fecal microbiota transplants from AD (FMT-AD) mice into young C57BL/6 (wild-type, WT) mice following TBI. Thus, FMT-AD and fecal microbiota transplants from healthy controls (FMT-young) were administered orally to young WT mice after the TBI occurred. We first determined the gut microbiota diversity and composition by analyzing fulllength 16S rRNA sequences from mouse fecal samples using the Oxford Nanopore MinION technology. We collected the blood, brain, and gut tissues for protein and immunohistochemical analysis. Our results showed that FMT-AD treatment stimulates a higher relative abundance of Muribaculum intestinal and a decrease in Lactobacillus johnsonii compared FMT-young treatment in WT mice. Furthermore, WT mice exhibited larger lesion volumes, increased the number of activated microglia/macrophages cells, and reduced motor recovery after FMT-AD compared to FMT-young one day after TBI. Thus, the gut microbiota from AD mice not only aggravates the neuroinflammatory response and motor recovery, but also increases the lesion size after TBI in young WT mice.

Keywords: microbiome, traumatic brain injury, Alzheimer's Disease, fecal microbiota transplant, neuroinflammation. 


\section{Introduction}

Traumatic brain injury (TBI) has a devastating effect on the brain, causing both shortand long-term damage that is not effectively alleviated by traditional pharmacological treatments (Gruenbaum et al., 2016; McConeghy et al., 2012). The peak incidence of TBI occurs in younger adults and the elderly (Faul and Coronado, 2015). Of note, in older adults, TBI often results in an accumulation of neuroinflammation that can exacerbate the neuropathological changes and contribute to an increased risk and acceleration of dementia-related pathologies, including Alzheimer's Disease (AD) (Hinson et al., 2015; Shishido et al., 2019). Further, older adults have more extended recovery periods compared to their younger peers because of different neuroinflammatory pathology, indicating a need for more targeted treatments (Cheng et al., 2018).

Numerous studies have shown that the gut microbiota plays a critical role in both brain function and cognition as well as the formation of bacterial amyloids (Megur et al., 2020). AD can weaken gastrointestinal barrier function and promote a proinflammatory phenotype involving the microbiota. The production of amyloid by bacteria or fungi in the gut microbiota may induce neurodegeneration and inflammation (Friedland, 2015). The human gut microbiome plays an essential role in immunity, and dysbiosis may lead to a proinflammatory state, thereby resulting in neuroinflammation. Colonic inflammation and intestinal barrier disruption have been found in about $70 \%$ of $A D$ patients, as suggested by elevated fecal calprotectin levels (Leblhuber et al., 2015). Moreover, AD patients have alterations in the gut microbiome, and an increase in gram-negative species like Bacteroidetes, and a positive correlation with cerebral spinal fluid amyloid and tau have been found (Vogt et al., 2017). Lipopolysaccharide (LPS), a pathogen-associated molecular protein (PAMP) present on the outer cell wall of gram-negative organisms, plays an important role in inflammation. LPS and bacterial amyloids synthesized by the gut microbiota can trigger immune cells by binding microglia through toll-like receptors (TLR), leading to an M1 phenotype and pro-inflammatory state, resulting in neuroinflammation and neuronal death. LPS is three times higher in AD patients' plasma compared to healthy controls (Zhang et al., 2009). Brain injury decreases bacterial diversity in young and old populations, and a less diverse gut microbiome increases susceptibility to brain damage and recovery length (Kigerl et al., 2016; Zhang et al., 2021). Numerous studies demonstrate that the risk of developing $A D$ is higher in patients who have previously suffered concussions or brain 
trauma (Gilbert et al., 2014; LoBue et al., 2017; Schofield et al., 1997). In fact, AD progresses more rapidly in those who have experienced TBI. AD is characterized by progressive cognitive and motor impairment associated with the accumulation of beta-amyloid $(A \beta)$ protein and tau protein deposition (Glenner and Wong, 1984). The accumulation of amyloid precursor protein (APP), $A \beta$, and pathological tau were increased after TBI in mice (Washington et al., 2014; Yang et al., 2015). AD also causes an increase in neuroinflammation and neuronal loss that is further exacerbated by TBI (Kokiko-Cochran et al., 2016). Therefore, inflammatory pathways could be a compelling therapeutic target to decrease the acceleration of neurodegeneration process after brain injury in AD patients. Furthermore, AD patients have worsened prognosis for recovery from $\mathrm{TBI}$, and it is necessary to design more effective treatments.

The inflammatory responses that occur in response to both AD and TBI extend past the brain and into the periphery, and they potentially include inflammation from the immune response in the gut (Wang and Kasper, 2014). The gastrointestinal (GI) tract and the brain make up the gut-brain axis through neural, immune, endocrine, and metabolic signaling pathways (Cryan and Dinan, 2012). A decrease in the diversity of gut microbiota has been shown to significantly increase toxic components in the bloodstream (Barko et al., 2018; Fernandez-Real et al., 2015) that may can arrive to the brain causing neuroinflammation. Also, many of the detrimental effects in the brain caused by aging results from gastrointestinal dysfunction, decreased microbiota diversity, and increased gut permeability (Montagne et al., 2015). Further, dysbiosis in the gut of aged individuals causes the release of lipopolysaccharides (LPS) and inflammatory cytokines that disrupt the blood-brain barrier and promote neuroinflammation and neuronal injury, leading to acceleration of neurodegeneration (Montagne et al., 2015). Microbiota dysbiosis cause a decrease of bacterial metabolites such as gamma-aminobutyric acid (GABA) and short-chain fatty acids (SCFAs) production (Bravo et al., 2011). These compounds have the potential to translocate from the mucosa to systemic circulation and affect brain function. For example, SCFAs are considered key mediators for microbiota-microglia interaction.

Clinical studies have reported a lack of diversity in the fecal microbiota of AD patients (Vogt and Finlay, 2017). For example, the microbiota of aged individuals with AD have lower levels of butyrate-producing bacteria (Rieder et al., 2017), which could lead to increased neuroinflammation and cognitive loss. Similarly, numerous studies show that AD can weaken the gastrointestinal barrier and promote a proinflammatory phenotype involving intestinal microbes (Kesika et al., 2021). Therefore, we suggest novel therapeutic strategies to restore the bacterial flora of $A D$ patients in order to reduce the inflammatory response that can be 
accelerated by brain damage. Fecal microbiota transplantation (FMT) is a procedure wherein the fecal matter of a healthy individual is transferred into a dysbiotic gut in order to restore intestinal flora. The therapeutic potential of FMT treatment has been shown in an AD mouse model to alleviate pathogenesis (Sun et al., 2019). Similarly, FMT rescued the changes in gut microbiota induced by TBI and reverted TBI-induced neurological deficits in rats (Du et al., 2021). FMT from healthy to $A D$ transgenic mice decreased $A \beta$ accumulation and phosphorylation of tau protein via regulating intestinal and systemic immune responses (Kim et al., 2020). FMT treatment holds a lot of promise to restore the neurological functions.

Still, little is known about the relationship between the gut microbiome post-brain trauma and the progress and acceleration of $A D$ pathology. Further, the mechanisms by which the gut microbiota affects AD brain pathology after TBI remain unclear. Therefore, in this study, our goal was to determine whether an FMT from AD mice could alter brain trauma outcomes through the gut-brain axis in wild-type (WT) mice after TBI. We demonstrated that fecal microbiota from AD mice transplanted into young mice after TBI exerts a detrimental effect on neurodegenerative processes and motor recovery. To our knowledge, this is the first study to detect how the gut microbiome from aged $A D$ mice manages to worsen inflammatory results in young mice. We hypothesized that FMT-AD would alter the gut microbiome, worsen neuroinflammation and recovery in injured WT mice. Our study may explain why recovery processes after brain injury are worse in $\mathrm{AD}$ patients and the need to design differentiated therapeutic strategies. 


\section{Material and Methods}

\subsection{Mice and traumatic brain injury model}

Young adult (9-12 weeks-old) male and female C57BL/6 mice were purchased from Jackson Laboratories (Bar Harbor, ME, USA). 3xTg-AD mice, 18-24 months old, were housed at the Houston Methodist Research Institute animal facilities. We performed qPCR-based genotyping from tail biopsies to confirm hemizygous and WT genotypes for the 3xTg-AD strain using the Transnetyx, Inc. (Cordova, TN) genotyping service. We used an electromagnetically controlled cortical impact $(\mathrm{CCl})$ injury device (Impact One impactor $\mathrm{CCl}$, Leica) to induce TBI in the cerebral cortex of C57BL/6 mice. As previously described by our laboratory (Villapol et al., 2015a), we applied the following impact coordinates: $2 \mathrm{~mm}$ lateral and $2 \mathrm{~mm}$ posterior to Bregma with an impact depth of $1.5 \mathrm{~mm}$, using a $2 \mathrm{~mm}$ diameter flat impact tip (speed $3.6 \mathrm{~m} / \mathrm{s}$, dwell time $100 \mathrm{~ms}$ ). Sham mice underwent all procedures except impact. Mice were sacrificed 3 days post-injury. Institutional Animal Care and Use Committee (IACUC) approved all animal studies and breeding protocols at Houston Methodist Research Institute. Studies were conducted following the NRC guide to the Care and Use of Laboratory Animals.

\subsection{Fecal microbiota transplantation (FMT), fecal sample collection, and DNA extraction}

Mice were group-housed based on their experimental group, and daily fecal samples from cages were pooled and redistributed amongst all experimental cages, thereby ensuring microbiome conformity through all mice. Fresh stool pellets were collected from aged 3xTg-AD donor mice for FMT. Next, the feces were homogenized in sterile phosphate buffered saline (PBS) and centrifuged at $800 \mathrm{~g}$ for 3 minutes to pellet the large particles. The supernatants were collected in fresh sterile tubes, and the final bacterial suspension was stored at $-80^{\circ} \mathrm{C}$. A single $200 \mu \mathrm{l}$ bolus of the prepared microbiota suspension was administered by oral gavage to each recipient C57BL/6 mice (wild-type, WT) at $24 \mathrm{~h}$ after TBI. Mice from the control group received a FMT from young WT mice. We used 5 mice/group with equal numbers of males and females to study sex differences. FMT recipient mice were sacrificed 3 days post-surgery, the abdomen cleaned with $70 \%$ ethanol, and the peritoneal cavity opened to expose the cecum. The cecal content was collected in sterile tubes for microbial DNA isolation and metagenomics analysis and stored at $-80^{\circ} \mathrm{C}$. Genetic material was isolated from frozen cecum samples using the QIAamp PowerFecal Pro DNA Kit (Qiagen, Germantown, MD). Bead beating was performed for $3 \mathrm{~min}$ at $6.5 \mathrm{~m} / \mathrm{s}$ on a FastPrep-24 system (MP Biomedicals, Irvine, CA). DNA isolation continued as specified in the kit's instructions. 


\subsection{Long read 16S rRNA gene sequencing}

16S rRNA amplicon sequencing was performed on a MinION nanopore sequencer (Oxford Nanopore Technologies, Oxford, UK). The amplicon library was prepared using the 16S Barcoding Kit 1-24 (SQK-16S024, Oxford Nanopore Technologies, Oxford, UK). For the PCR amplification and barcoding, $15 \mathrm{ng}$ of template DNA extracted from fecal samples were added to the LongAmp Hot Start Taq 2X Master Mix (New England Biolabs, Ipswich, MA). Initial denaturation at $95^{\circ} \mathrm{C}$ was followed by 35 cycles of $20 \mathrm{~s}$ at $95^{\circ} \mathrm{C}, 30 \mathrm{~s}$ at $55^{\circ} \mathrm{C}, 2$ min at $65^{\circ} \mathrm{C}$, and a final extension step of $5 \mathrm{~min}$ at $65{ }^{\circ} \mathrm{C}$. Purification of the barcoded amplicons was performed using the AMPure XP Beads (Beckman Coulter, Brea, CA) as per Nanopore's instructions. Samples were then quantified using a Qubit fluorometer (Life Technologies, Carlsbad, CA) and pooled in equimolar ratio to a total of 50-100 ng in $10 \mu$ l. The pooled library was then loaded into an R9.4.1 flow cell and run per the manufacturer's instructions. MINKNOW software 19.12.5 was used for data acquisition.

\subsection{Long-read $16 s$ sequencing data taxonomic bioinformatic analysis}

Raw sequences were base called with Guppy v4.4.2 with the trim barcodes function on and quality score (qscore) filtering set to a minimum score of 7 . Microbial community profiles for each sample were generated with Emu v1.0.2 (Curry et al., 2021), utilizing the default parameters and database. Alpha and beta diversity analyses for microbiome samples were performed with Python3.8 using the sci-kit bio v0.4.1 diversity package. Alpha diversity values were generated for each sample using the Shannon and Simpson indices, while beta diversity was calculated with the Weighted UniFrac metric. The phylogenetic tree was generated from the Emu default database using the taxdump_to_tree.py python script in biocore with each branch length set to 1. A principal coordinate analysis (PCoA) distance matrix was then generated, and statistical difference between the two groups was calculated with 9999 simulated ANOSIM permutations. To visualize these values, a Matplotlib v.3.3.3 beta diversity scatter plot was generated with 95\% confidence ellipses drawn around each group courtesy of Matplotlib twodimensional confidence ellipse source code. Stacked bar plots were created with GraphPad Prism version 9 to visualize the average relative abundance of bacteria amongst samples in each group for the given workflow. Log2 fold change was calculated between two samples from the same participant by taking the log2 of the percent relative abundance of the later time point divided by an earlier time point. To avoid mathematical errors, relative abundance values of 0 were transformed to $10^{-10}$. 


\subsection{Rotarod}

Motor performance was assessed by the ability of mice to stay on a rotating rod apparatus (Ugo Basile Harvard Apparatus, PA, USA) as the speed gradually increased. Mice were tested 2 days before injury (baseline) and one-day post-injury. The rod was accelerated from 4 to $40 \mathrm{rpm}$ in 2 $\mathrm{min}$, and the duration that mice were able to stay on the rod was recorded as latency to fall in seconds.

\subsection{Cresyl violet staining and lesion measurements}

Brains were fixed in $4 \%$ paraformaldehyde overnight and then stored in a $30 \%$ sucrose solution at $4^{\circ} \mathrm{C}$. Next, brains were sectioned at $20 \mu \mathrm{m}$ thickness in coronal orientation through the dorsal hippocampus. The brain sections were cryoprotected in an antifreeze solution (30\% glycerol, $\square 30 \%$ ethylene glycol in $0.01 \mathrm{M} \mathrm{PBS})$ for storage at $-20^{\circ} \mathrm{C}$. Cresyl-violet $(0.1 \%$, SigmaAldrich) was dissolved in distilled water and filtered. Brain sections were mounted on gelatincoated glass slides (SuperFrost Plus, Thermo Fisher Scientific, IL) and stained for 20 min with a cresyl-violet solution. Sections were then dehydrated for 2 min sequentially with 100, 95, 70, then $50 \%$ ethanol, cleared in xylene for another 2 min, covered with Permount mounting media (Fisher Scientific), and coverslipped. The lesion area was assessed on 8 to 12 brain sections spaced equidistance (every $450 \mathrm{~mm}$ ) apart, approximately -1.70 to $-2.70 \mathrm{~mm}$ from Bregma. Lesion volume was obtained by multiplying the sum of the lesion areas by the distance between sections. The percent of lesion volume was calculated by dividing each lesion volume by the total ipsilateral hemisphere volume (similarly obtained by multiplying the sum of the areas of the ipsilateral hemispheres by the distance between sections). Lesion scores ranged from 0 to 4 ( 0 , no lesion; 1, small cortical lesion; 2, medium cortical lesion; 3, large cortical lesion and small hippocampal lesion; 4, large cortical and hippocampal lesion).

\subsection{Immunofluorescence analysis}

Free-floating parallel brain sections were washed with PBS $+0.5 \%$ Triton X-100 (PBS-T) and then blocked with 5\% normal goat serum (NGS, \#S1000, Vector laboratories, Burlingame, CA) in PBS-T for $1 \mathrm{~h}$. Brain sections were incubated at $4{ }^{\circ} \mathrm{C}$ overnight in PBS-T and $3 \%$ of NGS using the following primary antibodies: anti-rabbit lba-1 (1:1000, Wako) for activated microglia/macrophages, anti-mouse GFAP for astrocytes (1:1000, Millipore) or anti-rat Ly-6B.2 alloantigen (1:100, MCA771GA, Bio-Rad Laboratories, Hercules, CA) for neutrophils. After incubation, brain sections were washed in PBS-T and incubated with the corresponding anti- 
rabbit or anti-mouse Alexa Fluor 568-conjugated and anti-rat Alexa Fluor 488-conjugated IgG secondary antibody (all 1:1000, Thermo Fisher Scientific, Waltham, MA) for $2 \mathrm{~h}$ at room temperature. Sections were washed with PBS and incubated with DAPI solution in PBS (1:50,000, Sigma-Aldrich, St. Louis, MO) for counterstained nuclei. The sections were rinsed with distilled water and coverslipped with Fluoro-Gel with Tris Buffer mounting medium (Electron Microscopy Sciences, Hatfield, PA). Images were acquired on a Nikon motorized fluorescence microscope (Eclipse Ni-U, Melville, NY) with a pco. Edge Scomos camera (4.2LT USB3) and analyzed using NIS-Elements software. For quantitative analysis of immunolabeled sections, we implemented unbiased, standardized sampling techniques to measure tissue areas corresponding to the injured cortex showing positive immunoreactivity, as we previously described (Villapol et al., 2015a; Villapol et al., 2014). To quantify the number of Ly6B.2-positive cells in the injured cortex, an average of four coronal sections from the lesion epicenter ( -1.34 to $-2.30 \mathrm{~mm}$ from Bregma) were counted and imaged for each animal, $\mathrm{n}=5$ mice per group. Within each brain region, every positive cell was counted in each of 5 cortical fields $(x 20,151.894$ $\mathrm{mm}^{2}$ ) around the impact area, as we have previously described (Villapol et al., 2017).

\subsection{Western blot analysis}

For serum collection, trunk blood was collected and allowed to clot for $30 \mathrm{~min}$ at room temperature. Next, serum was obtained by centrifugation $(1,500 \times \mathrm{xg})$ at $4{ }^{\circ} \mathrm{C}$ for $20 \mathrm{~min}$ and stored at $-80^{\circ} \mathrm{C}$. A $1: 5$ dilution from serum samples was combined with $4{ }^{\circ} \mathrm{C}$ Laemmli sample buffer (Bio-Rad Laboratories, Hercules, CA) and heated at $100{ }^{\circ} \mathrm{C}$ for 10 min before loading into 12\% Mini-PROTEAN TGX Stain-Free gels (Bio-Rad Laboratories, Hercules, CA). Separated proteins were transferred onto nitrocellulose membranes (Bio-Rad Laboratories, Hercules, CA). Following transfer, the membranes were blocked in 5\% w/v skim milk powder in PBS-Tween 20 (PBS-Tw) blocking buffer for $1 \mathrm{~h}$ at room temperature. Membranes were incubated with goat anti-SAA primary antibody (1:500; AF2948, R\&D Systems, Minneapolis, MN) overnight at $4{ }^{\circ} \mathrm{C}$ and with horseradish peroxidase-conjugated rabbit anti-goat secondary antibody (1:3000; Thermo Fisher Scientific, Waltham, MA) for $1 \mathrm{~h}$ at room temperature. Membranes were developed with Clarity Western ECL (Bio-Rad Laboratories, Hercules, CA). A ChemiDoc MP imaging system (Bio-Rad Laboratories, Hercules, CA) was used for the stain-free gel. The chemiluminescence imaging and densitometry quantification was performed using ImageLab 6.0.1 software (Bio-Rad Laboratories, Hercules, CA).

\subsection{Gut histological analysis}


Intestines from FMT-recipient mice were fixed in $4 \%$ paraformaldehyde for $48 \mathrm{~h}$ and transferred to $70 \%$ ethanol. Tissues were processed in a Shandon Exelsion ES Tissue Processor and embedded in paraffin on a Shandon HistoCenter Embedding System, using the manufacturer's standard processing and embedding protocols. Slides were sectioned at $5 \mu \mathrm{m}$ thickness. Paraffin sections were deparaffinized, rehydrated, and then stained with hematoxylin solution for $6 \mathrm{~h}$ at a temperature of $60-70{ }^{\circ} \mathrm{C}$. They were then rinsed in tap water until the water was colorless. Next, $0.3 \%$ acid alcohol in water was used to differentiate the tissue 2 times for 2 $\min$, and the sections were rinsed with tap water and stained with eosin for 2 min. Alcian blue stains the mucins produced by the goblet cells of the intestine. For Alcian Blue staining, deparaffinized and rehydrated sections were incubated in Alcian Blue solution for 30 min and counterstained with $0.1 \%$ Nuclear Fast Red for $5 \mathrm{~min}$. Sections were then dehydrated and mounted with permanent mounting media.

\subsection{Statistical analysis}

To analyze whether individual species or genus exhibited a significant difference in relative abundance between the FMT-AD and FMT-young group, we constructed linear models in MaAsLin2 (MaAsLin2 R package version 1.4.0, http://huttenhower.sph.harvard.edu/maaslin2). Relative abundance from Emu results were used as input, and default transformation and outcome distribution options were selected. No normalization methods were utilized since input data was already in relative abundance form. Models were run separately for species- and genus-level analysis. For both models, FMT donor was the fixed effect ( $n=9$ FMT-young, 8 FMT-AD), and a threshold for a relative abundance of at least $1 \%$ in a minimum of $10 \%$ of the samples was applied in order to reduce hypothesis tests requiring correction. For behavioral and immunostaining data analysis, we used two-way analysis of variance (ANOVA) followed by a Sidak posthoc test. An unpaired t-test was used to determine statistical differences in the gut histology quantifications. Data were represented as the mean and standard error of the mean $( \pm$ SEM), and $p$-values $<0.05$ were considered statistically significant. GraphPad Prism 9 software (Graph Pad Sofware Inc., CA, USA) was used for statistical analysis. All mice were randomized to experimental conditions, and experimenters were blind to genotype and treatment groups throughout the study. 


\section{Results}

\subsection{FMT-AD induced gut microbiome changes in C57BL/6 recipient mice following} TBI.

To assess the effect of $A D$ microbiota on recovery following $T B I$, we transplanted gut microbiota-derived from 3xTg-AD mice into young WT mice after brain injury. We prepared FMT from aged 3xTg-AD (FMT-AD) and young C57BL/6 WT (FMT-young) mice. Young WT male and female animals received their designated FMT-young or FMT-AD oral gavage after TBI (Figure 1). At 3 days post-injury, microbial DNA was extracted from cecum samples of the recipient mice, and next generation 16S rRNA gene sequencing was performed using the Nanopore MinION platform. We investigated the changes in the gut microbiome composition of the mice that received FMT-young compared to FMT-AD at the phylum and family levels by representing their relative abundances in stacked bar plots (Figure 2a, b). Some shifts were observed th the phylum level on the FMT-AD mice, mainly a reduction in Firmicutes together with an increase in Bacteroidetes (Figure 2a). However, the Firmicutes/Bacteroidetes ratios of the FMT-young and FMT-AD groups did not significantly differ (Mann-Whitney test $p=0.139$, mean ( \pm SEM) FMTyoung $=118.69( \pm 39.40), F M T-A D=45.17( \pm 12.02))$. Further changes were evident at the family level in the FMT-AD recipients compared to FMT-young, including a decrease in Lachnospiraceae and Clostridiaceae paired with a relative increase in Eubacteriaceae, Oscillospiraceae, and Muribaculaceae (Figure 2b). At the genus level, Muribaculum ( $q=0.02$ ) significantly increased relative abundance in FMT-AD mice, and the species Lactobacillus johnsonii ( $q=0.13$ ) reduced relative abundance, albeit not significantly (Figure 2c). However, there was no significant difference between the groups (Figure 2d). Further, the alpha diversity at the species level based on the Simpson and Shannon metrics did not significantly differ between the time points ( $p=0.178$ and $p=0.425$, respectively) (Figure 2e). Collectively, these results provide evidence that the fecal microbiota of FMT-AD is different from that of FMTyoung, which led us to the hypothesis that this difference may affect the neuroinflammatory responses following TBI.

\subsection{Microbiota from AD mice aggravated the lesion size after TBI.}


To evaluate whether the gut microbiota from $A D$ mice has any effect during the acute phase after TBI in WT mice, we assessed the cortical lesion size using cresyl-violet stained brain sections. We found a significant increase in mean lesion volume $(p=0.022)$ when calculated as a percent of total ipsilateral hemisphere volume in FMT-AD mice compared to FMT-young mice at 3 days post-TBI. This was confirmed by a significant increase in lesion score $(p=0.002)$ on a scale from 0 to 4 in FMT-AD mice compared to FMT-young mice (Figure 3 a-c).

\subsection{Microbiota from AD mice impaired motor ability after TBI.}

Motor dysfunction is a major consequence of TBI, and several motor assessments have been performed using the CCl injury model (Villapol et al., 2015a; Zinger et al., 2021). To investigate whether fecal transplant affects motor outcomes, we employed a rotarod test to compare motor skills in mice that received FMT from $A D$ mice and those that received FMT from young animals. Our results indicate that regardless of sex, motor function was significantly impaired in the FMT-AD group compared to the FMT-young group at 1 day after injury $(p<0.001)$ (Figure $3 d)$.

\subsection{FMT-AD led to increased neuroinflammation.}

Glia activation in the injured brain is detrimental at 3 days post-TBI (Villapol et al., 2014). Therefore, we analyzed changes in the activation of microglia, astrocytes, and neutrophil infiltration by detecting Iba-1, GFAP, and Ly6B.2 expression, respectively (Figure 4). Our results show an overall higher inflammatory response after FMT-AD compared to FMT-young. Specifically, there was increased microglia and macrophage activation and astroglial activation, but no change in neutrophil infiltration. Quantification of activated cells showed a significantly higher percent area of tissue occupied by lba1- and GFAP-positive cells $(p=0.001, p=0.0498$, respectively), indicating a higher percentage of microglia and macrophages (lba-1) and astroglia (GFAP) (Figure 4a, b, d, e). Neutrophil infiltration (Ly-6B.2), as quantified by the number of cells per field, showed no significant change (Figure 4c, f).

\subsection{FMT-AD did not increase Serum Amyloid A (SAA) levels nor did it induce gut alterations.}

Previously, we have demonstrated that TBI can induce alterations in the bloodstream and peripheral organs (Zinger et al., 2021). Therefore, we analyzed intestinal inflammation to clarify further whether neuropathological changes after TBI are related to the gut environment. SAA is a pro-inflammatory acute-phase protein that increases in the bloodstream after TBI (Soriano et 
al., 2020; Villapol et al., 2015b). SAA can be produced by the gut and travel through blood vessels to other inflamed tissues, including the brain. We measured the SAA levels in serum at 3 days post-injury in the mice that received FMT-young or FMT-AD. Quantitative western blot analysis showed an increase of 2.5-fold in males after FMT-AD compared to FMT-young, but not increase in females. However, no significant differences were observed (Figure 5 a, b). As expected, SAA levels were increased in injured mice compared to sham mice $\left({ }^{* *} p<0.01\right)$.

To evaluate if FMT was tolerated in vivo, intestines were collected, washed, fixed, sectioned, and stained with hematoxylin and eosin and Alcian blue to evaluate tissue damage. When comparing the intestines from FMT-young and FMT-AD mice, no differences were observed in either the Alcian blue area or the goblet cells/villus ratio in the small intestine in both male and female mice (Figure $5 \mathrm{c}, \mathrm{d}$ ). 


\section{Discussion}

In this study, we demonstrate the significant, and detrimental, impact of transplanting fecal microbiota collected from aged AD mice compared to young mice in healthy control mice. To demonstrate the detrimental role of the microbiota of $A D$ mice in response to trauma, we evaluated the impact of FMT-AD compared to FMT-young in the recovery of the injured brain of young mice. We found that FMT-AD worsened lesion size and inflammatory response, and motor behavior deteriorated further after TBI. Despite this, we did not find a significant effect on intestinal damage or inflammation in the periphery in FMT-AD compared to FMT-young mice. Therefore, our results suggest that not only can the gut microbiome be modified by a stool transplant after TBI, but that it may be a good target for treatments related to $A D$, TBI, or both. Given the detrimental link between brain injury and AD, FMT could be a strategy to improve recovery from trauma and reduce peripheral or central inflammation that could, if left untreated, accelerate the pathology of $A D$.

Accumulating evidence from human and animal studies supports the hypothesis that gut microbiota plays an important role in brain function. In animal models of stroke and spinal cord injury, the composition of bacterial taxa has been shown to change in response to central nervous system injury (Houlden et al., 2016; Kigerl et al., 2016) and those changes can persist for at least four weeks (Kigerl et al., 2016). Additionally, our previous work has demonstrated a decrease in the abundance of Lactobacillus gasseri, Ruminococcus flavefaciens, and Eubacterium ventriosum, and an increase in the abundance of Eubacterium sulci and Marvinbryantia formatexigens at $24 \mathrm{~h}$ after TBI in mice (Treangen et al., 2018). Conversely, receiving FMT from dysbiotic post-stroke mice has been shown to result in aggravated brain inflammation, lesion volume, and functional deficits (Benakis et al., 2016), suggesting that the gut microbiome can exert its influence via modulation of the brain neuroinflammatory response.

The growing awareness of the influential role of the gut microbiome has led to the development of novel treatment strategies. Notably, FMT has recently been established as an exciting treatment in multiple areas as the composition of the intestinal microbiome affects different organs and, therefore, may be involved in the development of different diseases (Liu et al., 2020b; Lucarini et al., 2021). For example, changes in the gut microbiota have accelerated neurological disorders by changing the metabolism of tryptophan (Kelly et al., 2016). Another treatment option used to modulate the microbiota is the administration of probiotics. Multiple studies have illustrated improved memory and behavioral deficits in mice after they received 
probiotics (Du et al. 2021; Liu et al. 2020a). For example, administration of Bifidobacterium bifidum and Lactobacillus Plantarum reduced $A \beta$ accumulation, leading to enhanced cognitive function in an $A D$ rat model (Shamsipour et al., 2021). Moreover, it was described that Firmicutes decrease and Bacteroidetes increase in AD transgenic mouse models compared to age-matched wild-type mice (Dos Santos Guilherme et al., 2020). Our current results show a trend wherein the levels of Lactobacillus johnsonii are decreased in FMT-AD animals compared to their FMT-young counterparts (Figure 2c). These results are further supported by a recent study that shows probiotics increased various Lactobacillus, including specifically the Lactobacillus johnsonii species, which prevented intestinal permeability, modulated proinflammatory factors, and correlated with better behavioral results (Wang et al., 2020). Other studies have shown that probiotics may increase the gut microbiome diversity and increase the abundance of Bacteroides and Faecalibacterium (Liu et al., 2020a). It was also found than probiotics improved memory deficits, and reduce cerebral neuronal and synaptic injuries, and glial activation in a mouse model of memory deficits and cognitive impairment (Yang et al., 2020). Similarly, our current results show that the relative abundance of the genera, Muribaculum significantly increase in FMT-AD compared to FMT-young mice (Figure 2). Previous studies have observed that Muribaculum decreases in response to a Western diet (McNamara et al., 2021), and a decrease in Muribaculum and an increase in Lactobacillus has been shown to occur in a rodent model of Crohn's disease (Dobranowski et al., 2019). Moreover, recent work has found decreased levels of Eubacterium rectale in the stool of $A D$ patients (Cattaneo et al., 2017) and in a work from our lab of cohort of athletes of contact sports following a concussion (Soriano et al., 2020). Eubacterium rectale is associated to inflammatory process.

The gut microbiome plays an important role in immune and inflammatory responses. Of note, it appears to be influential in neurological disorders, especially those related to cognitive problems, anxiety, or depression (Cryan and Dinan, 2012). We also know that intestinal microbial dysbiosis can lead to impaired inflammatory signaling, which has been suggested as a risk factor for brain recovery. In one study using a mouse model of cerebral ischemia, it was demonstrated that stroke causes intestinal dysbiosis and a proinflammatory response. Specifically, FMT of a healthy gut microbiome was able to reduce neuroinflammation and other functional deficits caused by stroke (Yu et al., 2021). Our study confirms the participation of the brain-gut-microbiome axis in inflammation, as we found a significant increase in glial activation (Figure 4). We saw greater activation and quantity of microglia, macrophages, and astroglia 
after FMT-AD in young mice. Another study on inflammation and the gut microbiome found that eradication and limitation of the complexity of the gut microbiota changed the properties of the microglia, specifically their maturation, differentiation, and function (Erny et al., 2015).

The impact of the gut microbiome can extend past neurological conditions to capacity and behavioral outcomes. Specifically, a recent study showed that FMT rescued bacterial and behavioral changes after TBI (Wang et al., 2020). As expected, we found that the microbiota of AD mice transplanted into young mice after TBI produced motor deficits (Figure 3d). Similarly, another study showed that intestinal dysbiosis affects the recovery of motor function after spinal cord injury (Kigerl et al., 2016). In this work, we did not find any significant change in peripheral inflammation or intestinal damage in mice after FMT-AD treatment; however, there was a tendency to increase. A lower number of animals per group may be the reason that these results are not significant at the three-day time point after TBI. Another possible explanation is that the three-day post-injury timepoint was too long, and the differences occur at shorter times during the first 24 hours.

We acknowledge that this study has specific limitations concerning the colonization capacity of the transplanted bacteria in the host animals and how this is reflected in response to brain trauma. Still, we believe that proper study of these variables would require long-term studies that are beyond those performed days after TBI. Additionally, to obtain a more translational analysis, microbiota from human $A D$ patients could be transplanted to mice and responses could be analyzed. Although the neuropathological response to TBI would continue to be in mice, characterizing specific bacteria and bacterial relationships identified in both animal and human clinical studies may lead to better interpretation of results. Consequently, we suggest that FMT from healthy donors to $A D$ patients suffering from $T B I$ could reduce $T B I$ neuroinflammation by remodeling the gut microbiota composition. Despite these limitations, our findings demonstrate that it is possible to reproduce the effects of the microbiota derived from $A D$ mice by transferring the fecal microbiota into young mice. Therefore, our findings suggest that AD gut microbiota alterations may play a critical role in the mechanisms underlying brain recovery. In this sense, the regulation of brain inflammation through the transplantation of the microbiota of healthy and young mice may be a therapeutic strategy for TBI associated to AD. Future experiments in our laboratory are oriented in this direction. 


\section{Conclusion}

Our study provides novel insight into the microbiota function in the neuropathology following brain injury. We demonstrated how the gut microbiota from AD mice aggravates the TBI outcomes, supporting the idea that the diversity and composition of the gut microbiome affects the impact of and recovery from neuroinflammatory disorders, specifically brain trauma in a preclinical mouse model. It provides strong support for the hypothesis that microbe-based approaches that aim to restore youth-like microbiota could improve cognitive function and improve recovery after brain damage. In the future, it will be interesting to see if restoration of the gut microbiota of $A D$ patients is also able to reduce the progression of brain damage. This represents a shift in $A D$ research, from the amyloid hypothesis to the microbial features underlying the disease. Understanding the microbiota effects in the context of the recovery of people with $A D$ or $A D$-associated dementia (an increasingly prevalent demographic segment of modern societies who also suffer head trauma) may have significant future implications for advancing their quality of life. Future research on brain-gut axis involvement in AD-associated $\mathrm{TBI}$ is necessary for new treatment targets and therapies.

\section{Author's contributions}

S.V. conceptualization, designed the study, and performed brain surgeries, S.S., K.C., Q.W., performed experiments, S.S., K.C., and S.V. analyzed data. S.S., K.C., E.C., T.T. and S.V. wrote the manuscript, and all the authors reviewed the manuscript

\section{Declaration of Competing Interest}

The authors have declared that no conflict of interest exists.

\section{Availability of data and materials}

The bacterial DNA-seq data has been deposited in the Rice University database under the accession number: GSE144125.

\section{Acknowledgments}

We thank Dr. Stephen Wong's laboratory at Houston Methodist for providing the 3xTg-AD mice for this study. We thank Ms. Shashi Krishnamurthy for her technical support in tissue processing. The authors are indebted to Dr. Gillian Hamilton for editing. Figure 1 was created using Biorender.com. This research was funded by an NIH grant R21NS106640 (SV) grant from the National Institute for Neurological Disorders and Stroke (NINDS) and Houston Methodist Research Institute. 
bioRxiv preprint doi: https://doi.org/10.1101/2021.11.23.469725; this version posted November 23,2021 . The copyright holder for this preprint

(which was not certified by peer review) is the author/funder, who has granted bioRxiv a license to display the preprint in perpetuity. It is made available under aCC-BY-NC-ND 4.0 International license.

\section{List of Abbreviations:}

Ab: amyloid-beta peptide

AD: Alzheimer Disease

APP: amyloid precursor protein

CCl: controlled cortical impact

GFAP: glial fibrilar astrocytic protein

FMT: fecal microbiota transplants

LPS: lipopolysaccharide

PBS: phosphate buffered saline

TBI: traumatic brain injury

TLR: toll-like receptors

WT: wild-type 


\section{FIGURE LEGENDS}

Figure 1. Experimental design. The controlled cortical injury (a model of TBI) was performed on WT mice. FMT-young and FMT-AD groups received fecal matter transplants by oral gavage from young WT and aged 3xTg-AD mice, respectively. Motor performance on the rotarod test was assessed before the injury and at 1 and 2-days post-TBI. Brains, blood, and colon samples were collected 3 days post-TBI for immunohistochemical and molecular characterization. Gut microbiome composition from cecal contents was analyzed by 16S rRNA sequencing using the Nanopore platform.

Figure 2. Gut microbiome changes after FMT from aged AD mice. (a-b) Relative abundances of the top 9 phyla (a) and families (b) in the cecum microbiota of mice that received FMT from young WT (FMT-young) or aged AD (FMT-AD) mice. (c) Relative abundances of the genera and species show significant alterations in the random-effects multivariate analysis for FMT-young ( $n=9$, gray) and FMT-AD ( $n=8$, red). An abundance cutoff of $>0.1 \%$ was applied at the species level. ${ }^{*} \mathrm{q}<0.05$. (d) Principal Coordinate Analysis (PCoA) ordination plot based on Weighted UniFrac distances for FMT-young ( $n=9$, gray) and FMT-AD ( $n=8$, red). The confidence ellipses represent the 95\% confidence interval. Analysis of Similarity (ANOSIM) evaluated group dissimilarities. (e) Shannon and Simpson alpha diversity indices at the species level for FMTyoung ( $n=9$, gray) and FMT-AD ( $n=8$, red). In the box and whisker plots, the cross represents the mean. Significance was determined by Kruskal-Wallis, followed by Dunn's multiple comparisons test.

Figure 3. Microbiota from AD mice aggravates lesion size and impairs motor ability after TBI. (a) Representative images (top) and sections stained with cresyl violet (bottom) of injured brains at 3 days post-injury. The dotted line indicates the lesion area composed of the cavity and edematous area. (b-c) FMT from AD mice significantly increased the mean lesion volume compared with lesion volume in mice that received FMT from young animals. $n=5$. ANOVA: ${ }^{*} \mathrm{p}<0.05,{ }^{* *} \mathrm{p}<0.01$ (ANOVA), ${ }^{*} \mathrm{p}<0.05$ (post-hoc test). (d) Rotarod motor test shows that FMT from AD mice further impaired motor ability 1-day post-TBI compared to FMT from young mice, $n=5 .{ }^{* *} p<0.001$. 
Figure 4. Microbiota from AD mice increased neuroinflammation in injured C57BL/6 mice. Glia activation increases in the injured brain after fecal matter transplants from AD mice (FMTAD) compared to FMT from young mice (FMT-young). Representative immunofluorescence images indicate that FMT-AD compared to FMT-young induces an increased inflammatory response in microglia/macrophages (Iba-1; a, a1-a4) and astroglia (GFAP; b, b1-b4) but no change in neutrophils (Ly-6B.2; c, c1-c4) at 3 days post-injury. The scale bar is $50 \mu \mathrm{m}$. The quantification graphs show a significantly higher area occupied by lba-1 (d) and GFAP (e) positive cells in FMT recipients from AD mice. (f) The number of cells Ly-6B.2 not significantly changed in the FMT-AD mice. $n=5$. ${ }^{*} p<0.05,{ }^{* *} p<0.01$ (ANOVA), ${ }^{\# \#} p<0.01$ (post-hoc test).

\section{Figure 5. FMT-AD neither induced peripheral inflammation nor caused intestinal damage} to recipient mice. (a) Western blot showing protein Serum Amyloid A (SAA) levels in the serum from FMT-AD and FMT-young (FMT-y) mice at 3-d post-TBI. (b) Quantification of the western blot shows a not statistically significant increase in SAA in the serum of males after FMT-AD compared to FMT-y. (c) Representative histology images from intestinal tissue do not show any abnormality between the FMT-y and FMT-AD groups in male or female mice. Left, Hematoxylin and eosin (H.E.) staining; right, Alcian blue staining. (d) Densiometric quantification of the Alcian blue positive area and counting the number of goblet cells per villus are not different between FMT-y and FMT-AD groups. 


\section{REFERENCES}

Barko, P. C., et al., 2018. The Gastrointestinal Microbiome: A Review. J Vet Intern Med. 32, 925.

Benakis, C., et al., 2016. Commensal microbiota affects ischemic stroke outcome by regulating intestinal gammadelta T cells. Nat Med. 22, 516-23.

Bravo, J. A., et al., 2011. Ingestion of Lactobacillus strain regulates emotional behavior and central GABA receptor expression in a mouse via the vagus nerve. Proc Natl Acad Sci U S A. $108,16050-5$.

Cattaneo, A., et al., 2017. Association of brain amyloidosis with pro-inflammatory gut bacterial taxa and peripheral inflammation markers in cognitively impaired elderly. Neurobiol Aging. 49, 60-68.

Cheng, W. H., et al., 2018. Age at injury and genotype modify acute inflammatory and neurofilament-light responses to mild CHIMERA traumatic brain injury in wild-type and APP/PS1 mice. Exp Neurol. 301, 26-38.

Cryan, J. F., Dinan, T. G., 2012. Mind-altering microorganisms: the impact of the gut microbiota on brain and behaviour. Nat Rev Neurosci. 13, 701-12.

Curry, K. D., et al., 2021. Emu: Species-Level Microbial Community Profiling for Full-Length Nanopore 16S Reads. bioRxiv 2021.05.02.442339.

Dobranowski, P. A., et al., 2019. Compositional changes to the ileal microbiome precede the onset of spontaneous ileitis in SHIP deficient mice. Gut Microbes. 10, 578-598.

Dos Santos Guilherme, M., et al., 2020. Impact of Acute and Chronic Amyloid-beta Peptide Exposure on Gut Microbial Commensals in the Mouse. Front Microbiol. 11, 1008.

Du, D., et al., 2021. Fecal Microbiota Transplantation Is a Promising Method to Restore Gut Microbiota Dysbiosis and Relieve Neurological Deficits after Traumatic Brain Injury. Oxid Med Cell Longev. 2021, 5816837.

Erny, D., et al., 2015. Host microbiota constantly control maturation and function of microglia in the CNS. Nat Neurosci. 18, 965-77.

Faul, M., Coronado, V., 2015. Epidemiology of traumatic brain injury. Handb Clin Neurol. 127, 313.

Fernandez-Real, J. M., et al., 2015. Gut Microbiota Interacts With Brain Microstructure and Function. J Clin Endocrinol Metab. 100, 4505-13.

Friedland, R. P., 2015. Mechanisms of molecular mimicry involving the microbiota in neurodegeneration. J Alzheimers Dis. 45, 349-62. 
Gilbert, M., et al., 2014. The association of traumatic brain injury with rate of progression of cognitive and functional impairment in a population-based cohort of Alzheimer's disease: the Cache County Dementia Progression Study. Int Psychogeriatr. 26, 1593-601.

Glenner, G. G., Wong, C. W., 1984. Alzheimer's disease: initial report of the purification and characterization of a novel cerebrovascular amyloid protein. Biochem Biophys Res Commun. 120, 885-90.

Gruenbaum, S. E., et al., 2016. Pharmacologic Neuroprotection for Functional Outcomes After Traumatic Brain Injury: A Systematic Review of the Clinical Literature. CNS Drugs. 30, 791-806.

Hinson, H. E., et al., 2015. Clinical evidence of inflammation driving secondary brain injury: a systematic review. J Trauma Acute Care Surg. 78, 184-91.

Houlden, A., et al., 2016. Brain injury induces specific changes in the caecal microbiota of mice via altered autonomic activity and mucoprotein production. Brain Behav Immun. 57, 1020.

Kelly, J. R., et al., 2016. Transferring the blues: Depression-associated gut microbiota induces neurobehavioural changes in the rat. J Psychiatr Res. 82, 109-18.

Kesika, P., et al., 2021. Role of gut-brain axis, gut microbial composition, and probiotic intervention in Alzheimer's disease. Life Sci. 264, 118627.

Kigerl, K. A., et al., 2016. Gut dysbiosis impairs recovery after spinal cord injury. J Exp Med. 213, 2603-2620.

Kim, S. M., et al., 2020. Fecal microbiota transplant rescues mice from human pathogen mediated sepsis by restoring systemic immunity. Nat Commun. 11, 2354.

Kokiko-Cochran, O., et al., 2016. Altered Neuroinflammation and Behavior after Traumatic Brain Injury in a Mouse Model of Alzheimer's Disease. J Neurotrauma. 33, 625-40.

Leblhuber, F., et al., 2015. Elevated fecal calprotectin in patients with Alzheimer's dementia indicates leaky gut. J Neural Transm (Vienna). 122, 1319-22.

Liu, J., et al., 2020a. High-altitude Tibetan fermented milk ameliorated cognitive dysfunction by modified gut microbiota in Alzheimer's disease transgenic mice. Food Funct. 11, 53085319.

Liu, S., et al., 2020b. Gut Microbiota and Dysbiosis in Alzheimer's Disease: Implications for Pathogenesis and Treatment. Mol Neurobiol. 57, 5026-5043.

LoBue, C., et al., 2017. Traumatic brain injury history is associated with earlier age of onset of Alzheimer disease. Clin Neuropsychol. 31, 85-98. 
Lucarini, E., et al., 2021. Visceral sensitivity modulation by faecal microbiota transplantation: the active role of gut bacteria in pain persistence. Pain.

McConeghy, K. W., et al., 2012. A review of neuroprotection pharmacology and therapies in patients with acute traumatic brain injury. CNS Drugs. 26, 613-36.

McNamara, M. P., et al., 2021. Early-life effects of juvenile Western diet and exercise on adult gut microbiome composition in mice. J Exp Biol. 224.

Megur, A., et al., 2020. The Microbiota-Gut-Brain Axis and Alzheimer's Disease: Neuroinflammation Is to Blame? Nutrients. 13.

Montagne, A., et al., 2015. Blood-brain barrier breakdown in the aging human hippocampus. Neuron. 85, 296-302.

Rieder, R., et al., 2017. Microbes and mental health: A review. Brain Behav Immun. 66, 9-17.

Schofield, P. W., et al., 1997. Alzheimer's disease after remote head injury: an incidence study. J Neurol Neurosurg Psychiatry. 62, 119-24.

Shamsipour, S., et al., 2021. Impact of interval training with probiotic (L. plantarum / Bifidobacterium bifidum) on passive avoidance test, ChAT and BDNF in the hippocampus of rats with Alzheimer's disease. Neurosci Lett. 756, 135949.

Shishido, H., et al., 2019. Traumatic Brain Injury by Weight-Drop Method Causes Transient Amyloid-beta Deposition and Acute Cognitive Deficits in Mice. Behav Neurol. 2019, 3248519.

Soriano, S., et al., 2020. Serum Amyloid A is Expressed in the Brain After Traumatic Brain Injury in a Sex-Dependent Manner. Cell Mol Neurobiol. 40, 1199-1211.

Sun, J., et al., 2019. Fecal microbiota transplantation alleviated Alzheimer's disease-like pathogenesis in APP/PS1 transgenic mice. Transl Psychiatry. 9, 189.

Treangen, T. J., et al., 2018. Traumatic Brain Injury in Mice Induces Acute Bacterial Dysbiosis Within the Fecal Microbiome. Front Immunol. 9, 2757.

Villapol, S., et al., 2015a. Neurorestoration after traumatic brain injury through angiotensin II receptor blockage. Brain. 138, 3299-315.

Villapol, S., et al., 2014. Temporal dynamics of cerebral blood flow, cortical damage, apoptosis, astrocyte-vasculature interaction and astrogliosis in the pericontusional region after traumatic brain injury. Front Neurol. 5, 82.

Villapol, S., et al., 2015b. Hepatic expression of serum amyloid A1 is induced by traumatic brain injury and modulated by telmisartan. Am J Pathol. 185, 2641-52.

Villapol, S., et al., 2017. Sexual dimorphism in the inflammatory response to traumatic brain injury. Glia. 65, 1423-1438. 
Vogt, N. M., et al., 2017. Gut microbiome alterations in Alzheimer's disease. Sci Rep. 7, 13537.

Vogt, S. L., Finlay, B. B., 2017. Gut microbiota-mediated protection against diarrheal infections.

J Travel Med. 24, S39-S43.

Wang, H., et al., 2020. Lactobacillus johnsonii BS15 Prevents Psychological Stress-Induced Memory Dysfunction in Mice by Modulating the Gut-Brain Axis. Front Microbiol. 11, 1941.

Wang, Y., Kasper, L. H., 2014. The role of microbiome in central nervous system disorders. Brain Behav Immun. 38, 1-12.

Washington, P. M., et al., 2014. Experimental traumatic brain injury induces rapid aggregation and oligomerization of amyloid-beta in an Alzheimer's disease mouse model. $\mathrm{J}$ Neurotrauma. 31, 125-34.

Yang, S. T., et al., 2015. Accumulation of amyloid in cognitive impairment after mild traumatic brain injury. J Neurol Sci. 349, 99-104.

Yang, X., et al., 2020. Probiotics modulate the microbiota-gut-brain axis and improve memory deficits in aged SAMP8 mice. Acta Pharm Sin B. 10, 475-487.

Yu, X., et al., 2021. Gut Microbiota Dysbiosis Induced by Intracerebral Hemorrhage Aggravates Neuroinflammation in Mice. Front Microbiol. 12, 647304.

Zhang, R., et al., 2009. Circulating endotoxin and systemic immune activation in sporadic amyotrophic lateral sclerosis (sALS). J Neuroimmunol. 206, 121-4.

Zhang, Y., et al., 2021. Gut microbiota-brain interaction: An emerging immunotherapy for traumatic brain injury. Exp Neurol. 337, 113585.

Zinger, A., et al., 2021. Biomimetic Nanoparticles as a Theranostic Tool for Traumatic Brain Injury. Adv Funct Mater. 31, 2100722. 


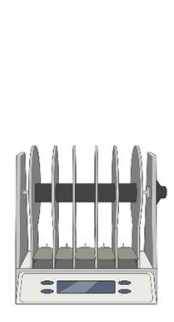

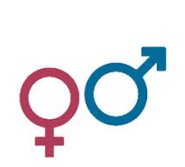

$-1 d$

$$
-2 d
$$

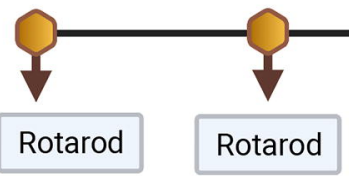

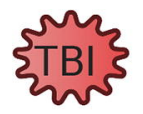

C57BL6

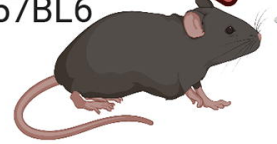

0
Lesion Volume Glia activation Neutrophil infiltration

Peripheral inflammation

FMT-young

FMT-AD

$+1 d$

$+3 d$

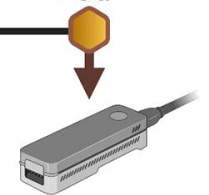

Nanopore Sequencing 

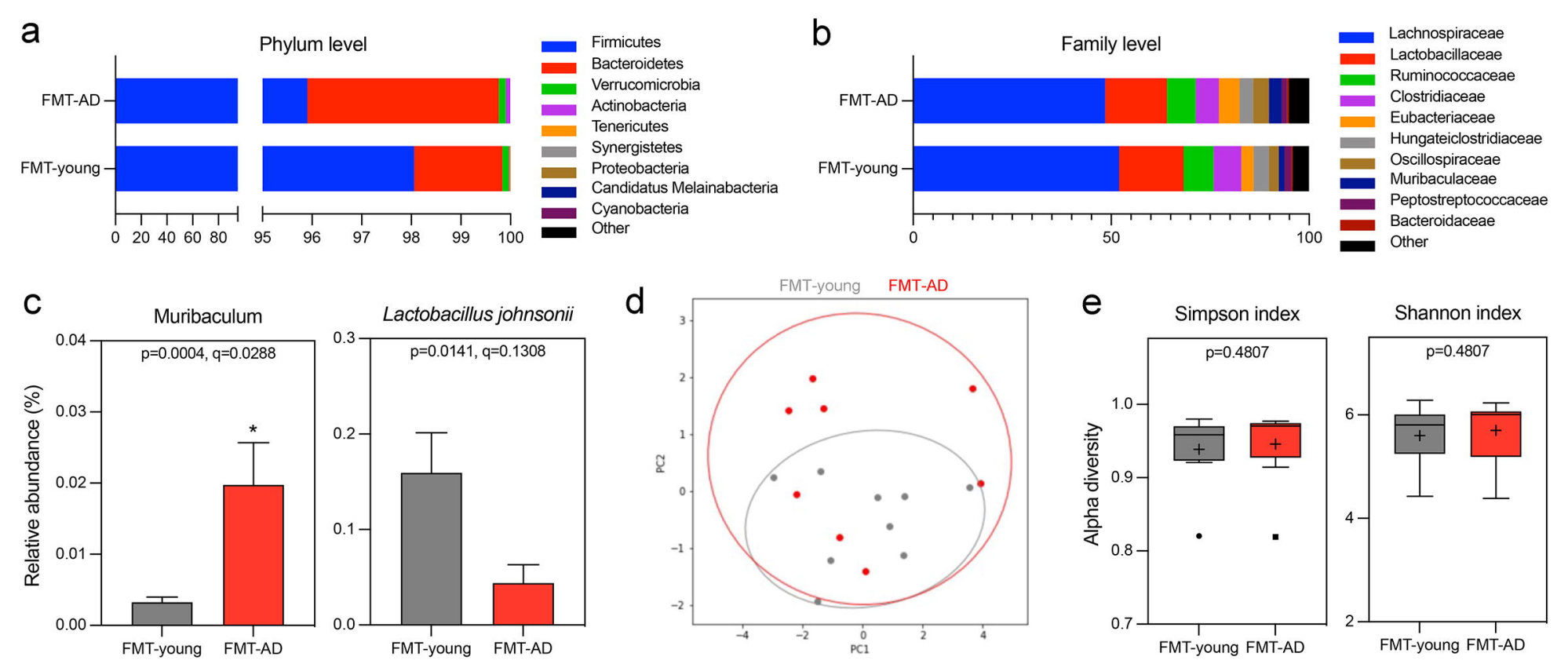
a

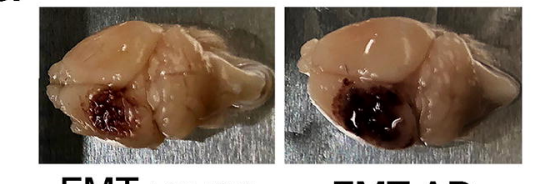

FMT-young FMT-AD

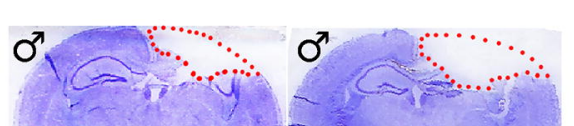

우요
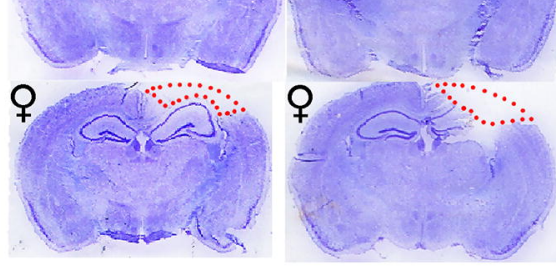

d

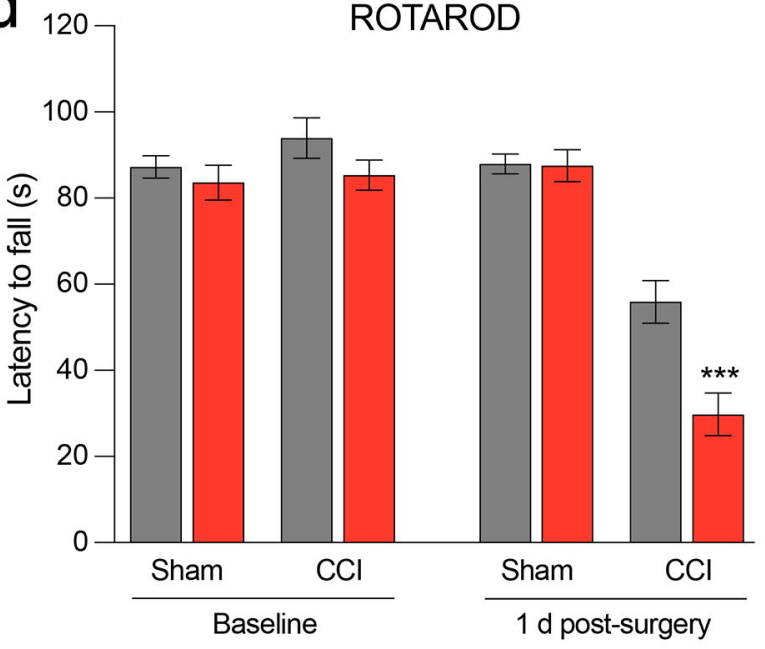

C
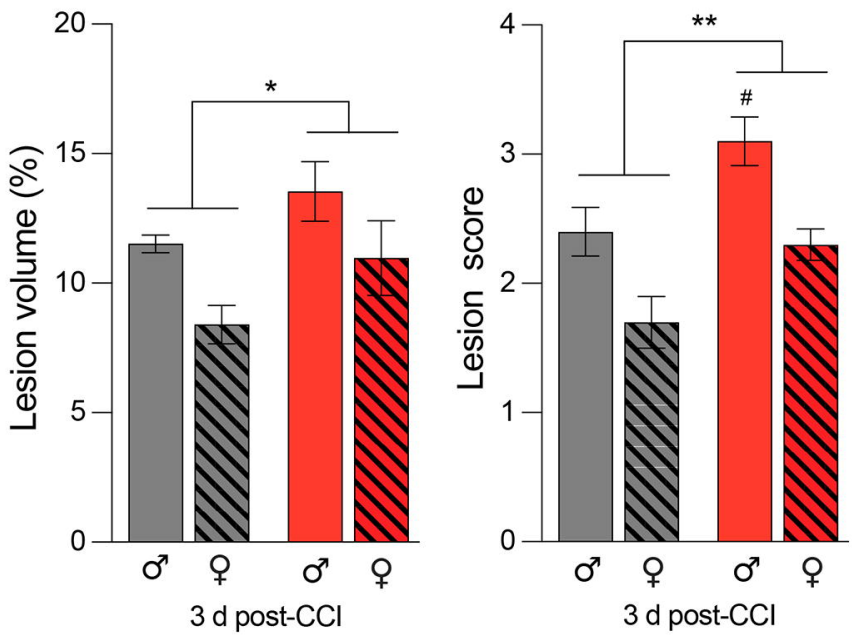

FMT-young

$\square$ FMT-AD 


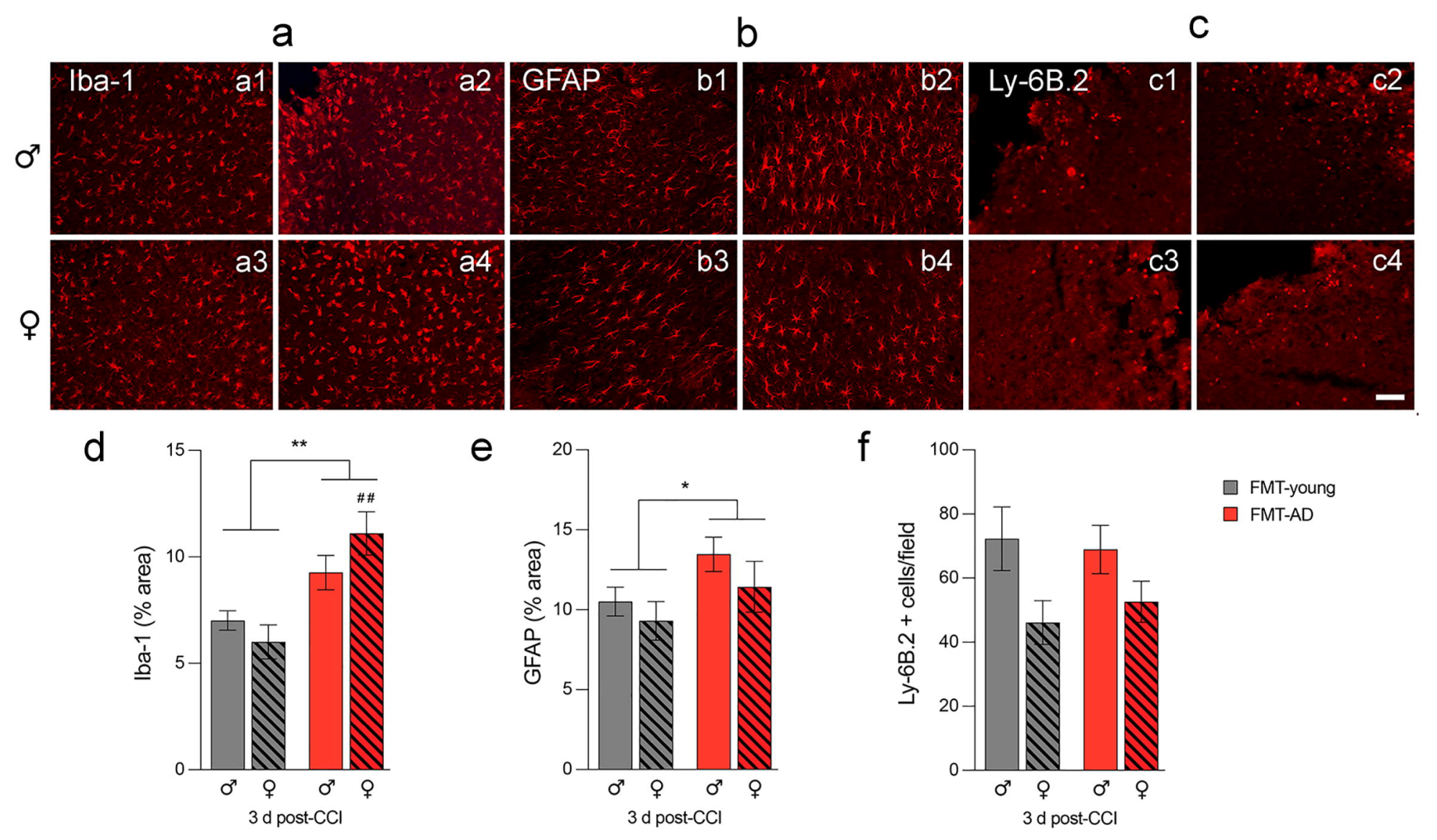


$a$

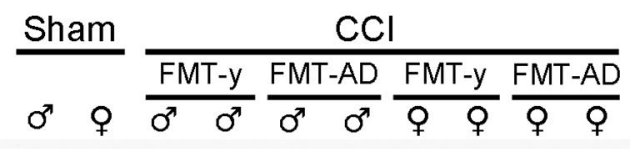

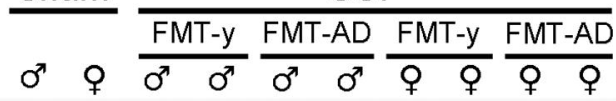

SAA
$(12 \mathrm{kDa})$

Stain

Free

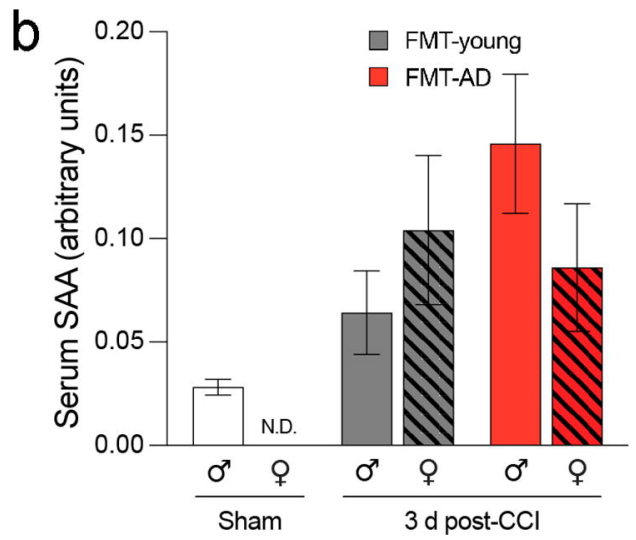

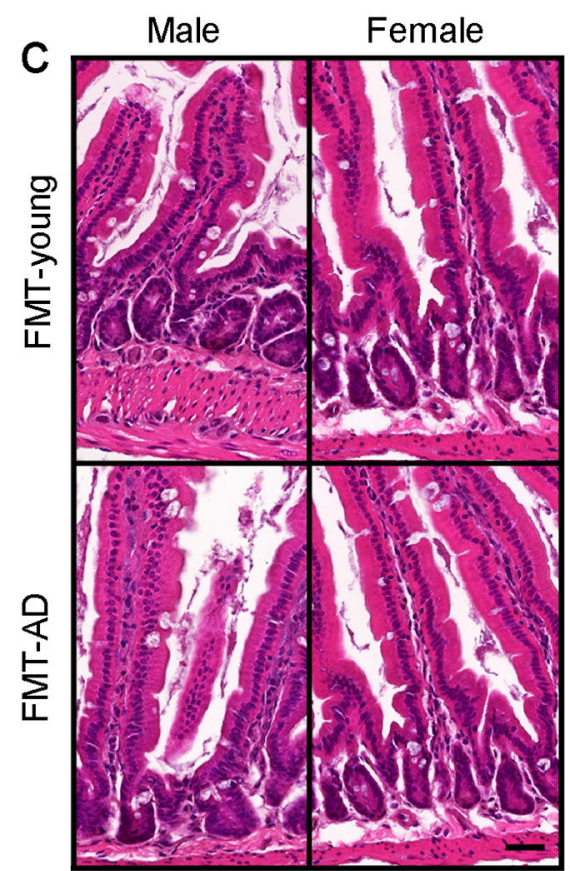

H.E. $x 10$

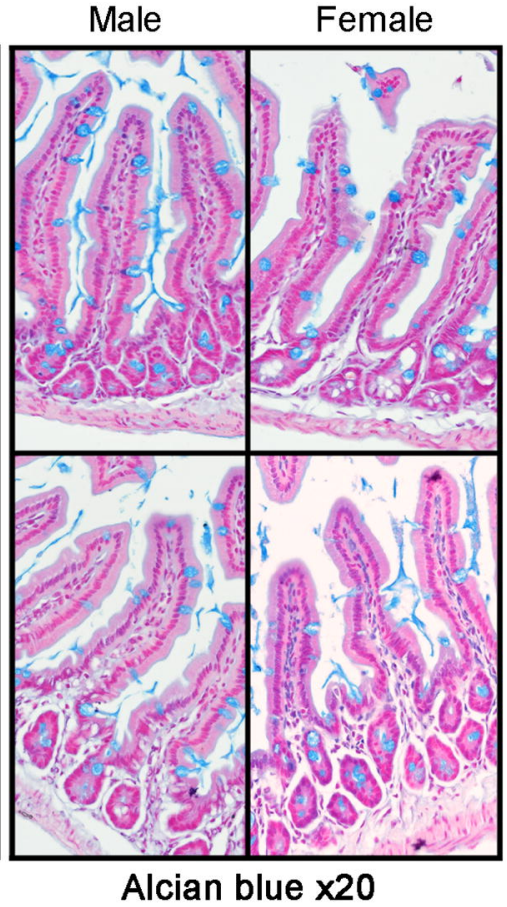

d

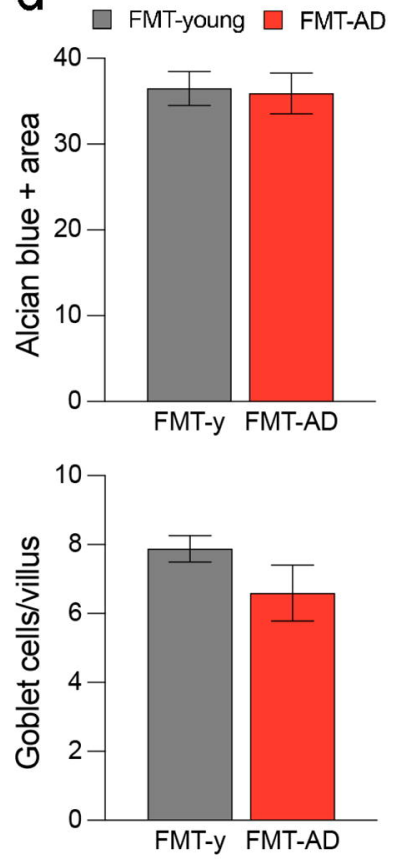

